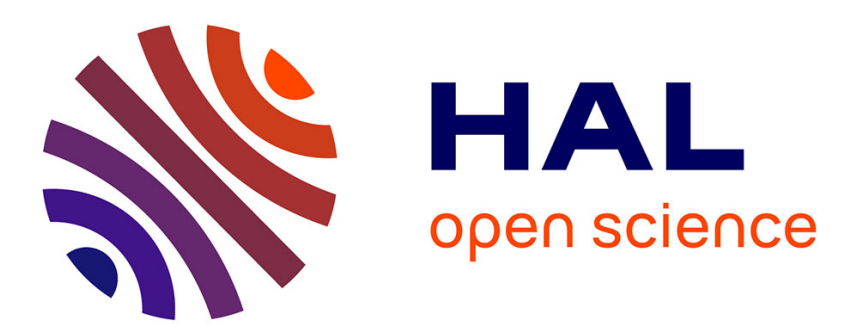

\title{
Migrants, transporteurs et agents de l'État: rencontre sur l'axe Agadez-Sebha
}

\author{
Julien Brachet
}

\section{To cite this version:}

Julien Brachet. Migrants, transporteurs et agents de l'État: rencontre sur l'axe Agadez-Sebha. Autrepart - Revue de sciences sociales au Sud, 2005, 36, pp.43-62. hal-00324190

\section{HAL Id: hal-00324190 https://hal.science/hal-00324190}

Submitted on 24 Sep 2008

HAL is a multi-disciplinary open access archive for the deposit and dissemination of scientific research documents, whether they are published or not. The documents may come from teaching and research institutions in France or abroad, or from public or private research centers.
L'archive ouverte pluridisciplinaire HAL, est destinée au dépôt et à la diffusion de documents scientifiques de niveau recherche, publiés ou non, émanant des établissements d'enseignement et de recherche français ou étrangers, des laboratoires publics ou privés. 


\title{
MigRANTS, TRANSPORTEURS ET AGENTS DE L'ÉTAT : RENCONTRE SUR L'AXE AGADEZ-SEBHA
}

Julien Brachet, Université Paris 1 Panthéon-Sorbonne (UMR Prodig), IRD (UR105).

Le Niger est traversé par d'importants flux migratoires en provenance d'Afrique subsaharienne et à destination de la Libye et de l'Europe. Ces flux, dont l'essor remonte au début des années 1990, se sont concentrés sur l'axe Agadez-Sebha, à la suite de l'ouverture officielle de la frontière terrestre nigéro-libyenne, faisant de cet axe l'une des principales voies de passage des migrations transsahariennes. Le Niger et la Libye, respectivement pays d'émigration et pays d'immigration, sont alors également devenus des pays de transit ${ }^{1}$. De véritables réseaux migratoires s'y sont développés, conjointement support et reflet de ces circulations humaines.

Dans une optique générale de compréhension du système migratoire euro-africain et de connaissance des formes d'inscriptions socio-spatiales des flux de transit migratoires au Sahara, nous proposerons ici une analyse de l'organisation de ces réseaux et des relations qu'entretiennent entre eux les divers groupes d'acteurs privés et publics qui les animent. A travers l'implication généralisée des agents de l'Etat nigérien dans ces circulations illégales, il s'agira de percevoir l'évolution des logiques de l'Etat et de son rapport à la loi qu'il édicte ${ }^{2}$.

\section{D'UNE RIVE À L'AUTRE DU SAHARA : ESPACES, TEMPS ET MODALITÉS DE LA} CIRCULATION MIGRATOIRE

\author{
"Que les uns d'entre vous ne mangent pas les biens des autres \\ illégalement. Mais qu'il y ait du négoce entre vous, par consentement \\ mutuel." \\ Extrait du Coran - Sourate 4, verset 29.
}

\footnotetext{
${ }^{1}$ «La Libye est aujourd'hui, pour les immigrés issus de l'Afrique subsaharienne, le principal pays de transit vers l'Europe... » (Le Monde, 05.06.2005).

${ }^{2}$ Cette étude s'appuie sur des travaux empiriques réalisés dans cette zone (Agadez, Toureyet, Bilma, Dirkou, Ségédine...) entre 2003 et 2005. Les entretiens cités au cours de cet article furent réalisés par l'auteur en français et en anglais, langues véhiculaires au sein du système migratoire étudié. Donnant lieu à des enregistrements sur micro cassettes, ces entretiens libres et parfois semi-directifs furent généralement précédés de plusieurs rencontres informelles entre l'auteur et les acteurs concernés.
} 


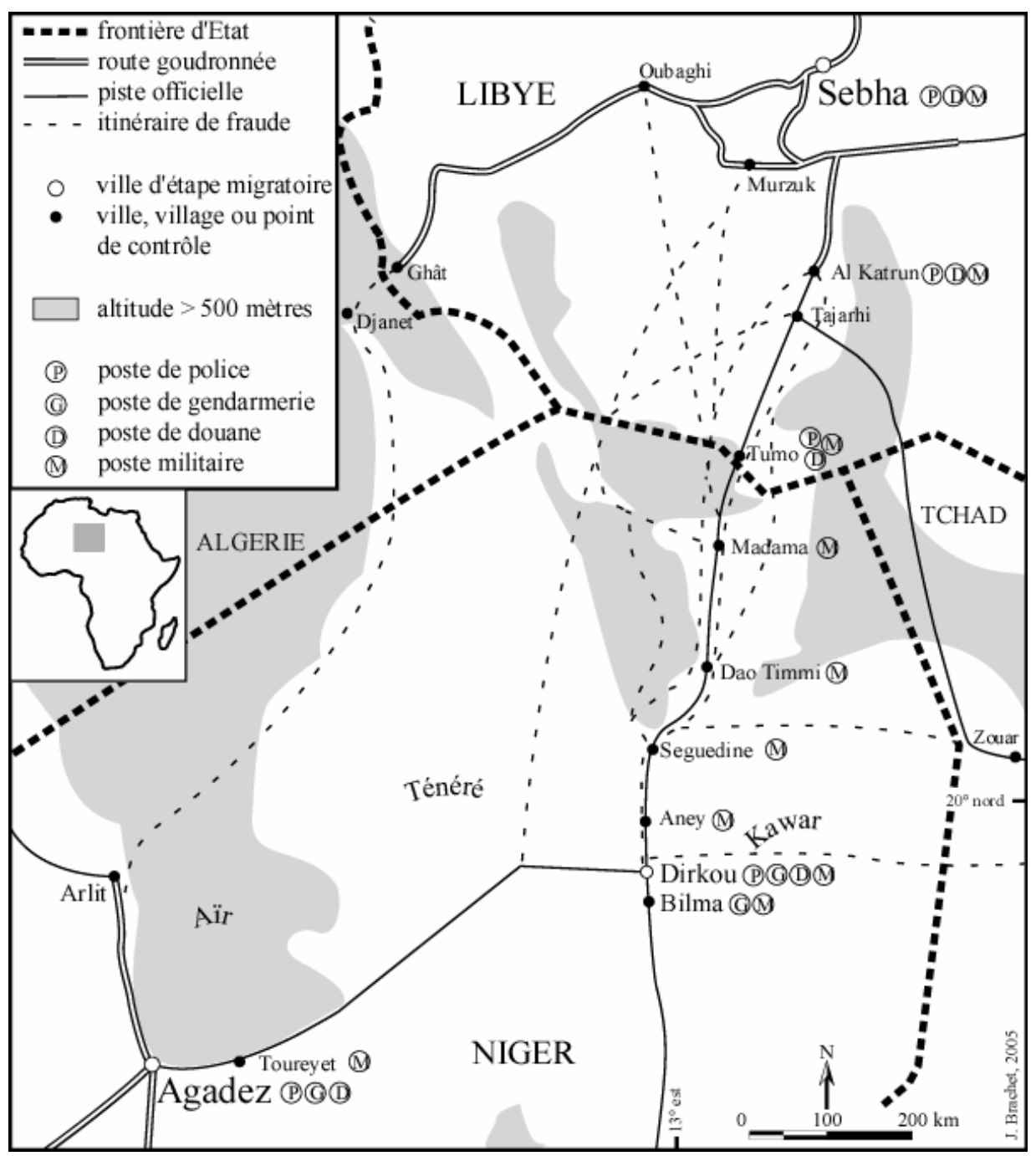

Fig. 1 - Agadez-Sebha : un itinéraire sous contrôle, des itinéraires sans contrôle.

Les principaux acteurs du système migratoire saharien, à savoir les migrants, les professionnels du transport et les agents des Etats, se rencontrent en divers endroits et moments des parcours afin de s'entendre sur les modalités pratiques et financières de ces mobilités. Leur mise en relation passe par de longues négociations, sans cesse renouvelées, et dont l'aboutissement est une circulation d'argent d'un individu à l'autre, d'un groupe à l'autre. Des lieux se créent ainsi dans la succession des rencontres, le long des itinéraires de migrations. Simples étapes ou véritables carrefours migratoires, ces lieux dévolus à l'accueil et au transport des migrants sont au cœur de l'organisation spatiale des villes et villages où ils se trouvent et constituent, à plus petite échelle, des éléments centraux de la structure de l'espace migratoire saharien. 


\section{Le carrefour migratoire agadézien au centre d'un système de transport spécialisé}

Agadez, jadis grand caravansérail, ville de négoce et de rencontre entre les populations du nord et du sud du Sahara, avait perdu de son dynamisme «depuis le déclin des échanges transsahariens et l'enclavement de la région septentrionale du Niger déterminé par les frontières coloniales » [Guitart, 1992 : 247]. L'avantage de position que lui confèrent les infrastructures contemporaines de transport (dernière ville nigérienne de l'axe Niger-Libye reliée au réseau routier bitumé - cf. Fig.1) et la présence en son sein de populations dont le capital culturel et social facilite la mobilité au Sahara central (populations arabes et touarègues), ont permis à Agadez de profiter du renouveau des migrations transsahariennes pour renouer avec la fonction de carrefour.

Des flux de migrants venus d'Afrique de l'Ouest et d'Afrique Centrale s'y rejoignent, s'y rencontrent, s'y mêlent. Des flots d'informations y circulent, à propos du désert et de ses dangers, de la meilleure manière de le traverser, des véhicules à prendre ou à éviter, des itinéraires à suivre et du passage des frontières, ou encore de la vie au Maghreb et en Europe. Ces échanges d'informations entre migrants en partance ou de retour, et entre migrants et autochtones, en un lieu de rencontre situé au croisement de plusieurs routes de directions méridiennes, incitent les migrants à ré-interroger leurs projets migratoires. Les possibilités qui s'offrent à eux créent ainsi une situation où le choix est de nouveau possible, et même nécessaire. Mais si la modification des objectifs de départ ou des itinéraires initialement prévus peut résulter d'un choix volontaire de la part des migrants, il arrive également que ces changements soient induits par des situations politiques conjoncturelles (fermeture de frontière, expulsions...).

La ville, travaillée par les flux de population qui la traverse, voit son paysage urbain se transformer. Certains de ses quartiers vivent ainsi au rythme des arrivées et des départs de migrants, tantôt animés par une foule cosmopolite, tantôt baignés par le calme pesant de l'attente. Tel est le cas du quartier de la gare routière d'Agadez où de nombreuses activités liées à la circulation migratoire se sont créées (structures d'hébergement, change de monnaie, vente de marchandises servant à la traversée du désert, agences de transport, lieux de prostitution...).

Au cœur du dispositif permettant le passage des migrants d'une rive à l'autre du désert se trouvent des agences de transport spécialisées. Douze sont officiellement enregistrées à la mairie d'Agadez en tant qu'"agences de courtage", mais dix étaient réellement en activité début 2005. Malgré le caractère équivoque de cette activité au regard de la loi, la création officielle d'une telle agence est rapide ("On peut faire ça en une ou deux journées" affirme un directeur d'agence) et peu onéreuse (coût d'une patente et de deux ou trois mois de loyer d'avance pour le bureau $-5 \mathrm{~m}^{2}$ - qui est octroyé dans l'enceinte de la gare routière). Les directeurs d'agence sont majoritairement originaires d'Agadez, mais également de Zinder, de Niamey et de Bilma. Leurs réseaux sociaux intègrent des acteurs privés (rabatteurs coxers-, passeurs-guide, chauffeurs, propriétaires de véhicules...) et publics 
(policiers, gendarmes, militaires...), qui organisent la mobilité des personnes dans l'ensemble du Nord du pays, et permettent donc l'exercice de cette activité au sein de la ville. Deux agences sont dirigées par des Nigériens nés et ayant vécu dans un pays d'émigration (Ghana et Nigeria) et qui y ont développé des réseaux qui leur permettent d'encadrer ces mouvements migratoires depuis leurs pays d'origine, ou tout au moins d'être le contact privilégié des migrants de ces pays à Agadez.

Dès leur arrivée à la barrière d'entrée de la ville, les migrants étrangers sont repérés par les policiers $^{3}$ qui les font descendre des véhicules, leurs prennent leurs papiers d'identité, et leur imposent le paiement d'une taxe dont le montant, négociable, va de quelques milliers à plus de 20000 francs CFA, sans que cela ne dépende directement de la régularité de leur situation. Ces pratiques de prélèvements par la police, bien que généralisées dans le transport routier en Afrique subsaharienne, semblent s'exercer bien davantage à l'encontre des voyageurs-migrants que des autres voyageurs.

Lorsque des migrants dont les papiers sont en règles refusent de payer, les policiers les gardent au poste de police et les mettent parfois en cellule jusqu'à ce qu'ils "acceptent" de débourser.

"Tout étranger qui passe à Agadez il faut qu'il paie l'argent... 5 000, 10000 , 15 000, jusqu'à 20000 même. [...] Parfois les étrangers ils viennent, ils n'ont pas assez d'argent... bon, ils nous expliquent que c'est en cours de route que la police a pris leur argent, parce qu'il y a beaucoup de barrières. Donc quand ils viennent ici à Agadez, leur argent c'est insuffisant pour continuer devant. Donc ça c'est un problème. Y'a d'autres qui ne peuvent pas retourner chez eux parce qu'ils ont abandonné leur travail, ils ont vendu ce qu'ils avaient... Y'en a qui restent à Agadez jusqu'à ce qu'ils mangent ce qu'ils ont dans leur main... Ils ne savent plus ce qu'ils font, c'est trop de souffrance... donc a un moment on s'est réunis, ceux qui ont des agences, et on est partis voir la police... Le commissaire de police a dit que maintenant il ne faut plus que la police prenne l'argent des étrangers, il a dit qu'il ne faut même plus faire descendre les étrangers à la barrière... mais jusqu'à présent la police prend l'argent des étrangers à partir de la barrière..." Adou, membre d'une agence de courtage - Agadez, le 29 novembre 2004.

Les policiers sont les premières personnes informées de l'arrivée des migrants dans la ville, lorsque ceux-ci passent par la voie normale. Afin d'obtenir rapidement ce renseignement, précieux au de la de concurrence,

\footnotetext{
3 "Les jeunes [migrants] tentés par l'eldorado libyen, qui transitent par le Niger sont facilement reconnaissables : filles et garçons s'habillent indifféremment en jeans, baskets et casquettes. Ils voyagent par groupes de plusieurs personnes. Ils ont des comportements, des gestes et de nombreux réflexes qui permettent aisément de les repérer. Dès que le car s'immobilise, ils en redescendent presque tous et s'assemblent aussitôt à l'écart des autres passagers. Ces marques de différenciation et ces réflexes de grégarité naturelle chez toute minorité, dans un espace géographique donné, ne facilitent malheureusement pas la traversée de l'étape nigérienne de la grande aventure de ces malheureux frères africains. De fait, ils s'exposent à de nombreuses tracasseries, ou du moins, ils sont littéralement livrés à des policiers qui n'ont d'autre grandeur, d'autre humanité ou d'autre divinité que l'argent." (Le Républicain, 15-21.01.2004 : 9).
} 
les directeurs d'agence offrent des cartes téléphoniques pour téléphones mobiles ${ }^{4}$ aux policiers en poste à la barrière qui, en contrepartie, s'engagent à les prévenir par téléphone à chaque arrivée de migrants afin qu'ils puissent envoyer leurs coxers ${ }^{5}$. Les policiers acceptent aussi fréquemment les dons simultanés de plusieurs directeurs d'agence, ce qui les oblige alors à appeler différentes personnes à la fois lorsque les migrants arrivent ou bien à n'en appeler qu'une au détriment des autres. Dans les deux cas, les tensions créées amènent de nouvelles négociations.

Les migrants sont conduit par des coxers jusqu'aux agences de voyage spécialisées où, quelle que soit leur destination, on leur promet systématiquement un départ dans les jours qui suivent, ce qui les incite à payer immédiatement leur billet et permet au directeur de l'agence de ne pas perdre ces clients au profit de ses concurrents. Les tarifs des trajets proposés par les agences varient selon divers facteurs (ouverture/fermeture des frontières, disponibilité et coût du carburant, nombre de passagers, capacité financière des passagers...) ; le tableau ci-dessous (fig. 2) expose les moyennes hautes et basses des tarifs de transport pour différents trajets en 2003-2004.

Fig. 2 - Tarifs des trajets proposés par les agences de transport d'Agadez et de Dirkou (en francs CFA, 2003-2004)

\begin{tabular}{|c|c|c|}
\hline Trajet & $\begin{array}{l}\text { Type de } \\
\text { véhicule }\end{array}$ & Tarif \\
\hline \multirow{2}{*}{ Agadez-Sebha } & camion & 40000 à 60000 \\
\hline & pick-up & 50000 à 80000 \\
\hline \multirow{2}{*}{ Agadez-Dirkou } & camion & 10000 à 20000 \\
\hline & pick-up & 20000 à 30000 \\
\hline \multirow{2}{*}{ Dirkou-Sebha } & camion & 30000 à 50000 \\
\hline & pick-up & 30000 à 40000 \\
\hline Dirkou-Gatrone & pick-up & $\begin{array}{l}30000 \text { à } 40000 \\
\text { Source : enquêtes 2003-2004. }\end{array}$ \\
\hline
\end{tabular}

Les sommes perçues par les directeurs d'agence sont réparties entre les différentes personnes qui participent à l'organisation et à la réalisation du transport. Par exemple, sur 50000 FCFA payés à l'agence par un passager, 5000 FCFA reviennent au coxer qui a amené le passager, 35000 FCFA sont pour le propriétaire du véhicule qui emmènera les migrants, et 10000 FCFA sont gardés par le chef d'agence. Les propriétaires des véhicules sont indépendants et ne travaillent que sur demande; c'est pourquoi, ils offrent fréquemment une ou deux places par voyage au directeur d'agence qui a fait appel à eux.

\footnotetext{
${ }^{4}$ Deux compagnies de téléphonie mobile se sont installées à Agadez en 2002.

${ }^{5}$ Terme qui désigne les rabatteurs chargés de trouver des passagers et de les ramener aux agences de transport.
} 
Les accords entre professionnels du transport et représentants de l'Etat sont nombreux. L'un des plus fréquents consiste à expliquer aux migrants détenteurs d'un carnet de voyage CEDEAO ou d'un passeport qu'ils doivent le faire viser par les services de police, pour ne pas avoir de problème jusqu'à la frontière, et que cette formalité coûte 1000 FCFA. Ceux qui le souhaitent remettent alors leur document de voyage à un membre de l'agence qui va se rendre au commissariat pour y faire apposer un tampon officiel. Ce tampon, superflu, sera négocié par la personne de l'agence en échange d'une partie seulement de la somme récoltée.

Ce type d'arrangement entre les membres des agences de transport et les policiers est symptomatique des logiques qui régissent les rapports entre migrants, professionnels du transport et représentants de l'Etat. Ces deux derniers groupes raisonnent dans des temporalités proches et ont conscience de leur commun intérêt. Ils s'entendent donc afin de tirer profit de la crédulité et de la vulnérabilité des migrants étrangers qui, eux, ne font que passer.

Cependant, en de rares cas, il arrive que des représentants de l'Etat soutiennent quelque peu les migrants victimes des pratiques abusives des professionnels du transport.

Un mois après avoir payé leur transport pour aller d'Agadez à Tamanghasset (40 000 FCFA), trois migrants camerounais sont toujours en attente à la gare routière d'Agadez. Le coût financier de cette attente imprévue les a appauvris. Le directeur de l'agence qui doit les faire voyager refuse de leur rendre leur argent alors que des véhicules circulant pour le compte d'autres agences sont déjà partis pour cette destination. Les trois migrants décident de porter plainte contre lui. Afin d'expliquer la situation aux policiers, ils se rendent alors au commissariat central et s'y font finalement emprisonner sous prétexte qu'ils n'ont pas de papiers en règles (alors qu'ils ont payé en arrivant à l'entrée d'Agadez aux mêmes policiers et pour cette même raison). Après quelques jours de cellule, ils paient 1000 FCFA pour être libérés. "On a compris que c'est un jeu où tout le monde gagne ici, les policiers, tout le monde... c'est un sale pays" résume Dieudonné (Agadez, le 31 décembre 2004). Plusieurs jours après, le directeur de l'agence n'accepte toujours pas de leur rendre leur argent ni de voyager. Les trois migrants vont rencontrer le procureur au palais de justice d'Agadez pour de nouveau porter plainte. Comprenant que ces voyageurs camerounais n'ont aucune alternative, n'ayant plus d'argent, le procureur accepte de saisir le commissaire de police afin qu'il règle ce contentieux. Le commissaire a alors demandé au directeur de l'agence d'envoyer rapidement les trois migrants à Tamanghasset, ce qui fut fait.

Carnet de terrain, extraits - Agadez, décembre 2004-janvier 2005.

Cet exemple de situation conflictuelle illustre le rapport qu'entretiennent les acteurs du système migratoire à la loi et à son application. Les migrants camerounais, en situation irrégulière sur le territoire nigérien, mais considérant être victime d'une injustice, n'ont pas hésité à aller à deux reprises se plaindre auprès des autorités nigériennes. Celles-ci ont alors rendu justice, tout d'abord en maintenant une relation d'intérêt avec le directeur d'agence, puis, en raison de la légitimité des requêtes, en prenant le parti des migrants. 
Lorsqu'une agence de transport a suffisamment de passagers pour une même destination, son directeur affrète un véhicule: soit un pick-up, qui transporte jusqu'à trente-deux passagers entassés sur son plateau arrière, soit un camion qui peut en emmener jusqu'à cent cinquante. Le chargement des véhicules se fait au sein de la gare routière, en présence de policiers. A chacun de ces départs officiels, les agences doivent remettre une "feuille de route" aux services de Police, qui spécifie l'immatriculation du véhicule, le nom du chauffeur et le numéro de son permis de conduire, ainsi que les noms et nationalités des passagers ; mais cette formalité est rarement remplie.

A la sortie d'Agadez, à deux kilomètres de la ville sur la voie de la Libye, les services de police procèdent à une nouvelle vérification des papiers des passagers, puis prélèvent une fois de plus une taxe non officielle qui va de 500 à quelques milliers de francs CFA. Le contrôle suivant a lieu 80 kilomètres plus loin, au poste de gendarmerie de Toureyet. Chaque migrant de passage, allant ou revenant de Libye, y est taxé d'environ 1000 FCFA $^{6}$.

\section{L'oasis de Dirkou, haut lieu du transit migratoire saharien}

Située au sortir du grand erg du Ténéré, à $650 \mathrm{~km}$ d'Agadez et $500 \mathrm{~km}$ de la frontière libyenne, le village de Dirkou constitue la principale étape des transporteurs qui circulent entre Agadez et Sebha. Le fort développement des mouvements migratoires entre le Niger et la Libye a donc engendré une transformation rapide de cette petite oasis du septentrion nigérien. En quelques années, la partie de la palmeraie où les migrants s'installaient de façon temporaire, en attendant de poursuivre leur voyage, est devenue un haut lieu du transit migratoire au Sahara, véritable quartier distinct de l'ancien village. De nombreuses activités s'y sont développées, liées directement au transport (autogare, agence de transport, garages, vente de carburants...) ou à la présence des migrants en transit (marché permanent bien achalandé, logements locatifs, bars, restaurants, "salons de coiffures", bureaux de change de monnaie, mais également poste de gendarmerie, de police et de douane), entraînant une croissance démographique du village sans précédent. Dénommé Sabon Gari, ce qui signifie «nouveau village » en hawsa, ce quartier est actuellement plus peuplé que le village d'origine, Gari n kawa ou «village du natron $»^{7}$.

\footnotetext{
${ }^{6}$ Peu avant les élections présidentielles de décembre 2004, à la suite des nombreuses plaintes de migrants nigériens, ce poste de gendarmerie a été supprimé et remplacé par un détachement militaire qui, officiellement, ne doit pas effectuer de contrôle.

${ }^{7}$ Cette différence de peuplement, remarquable à l'observation, ne peut être chiffrée du fait du manque d'exhaustivité du dernier recensement général de la population réalisé en 2001 et des variations notoires qui existent dans la restitution des résultats de ce recensement. D'après le cahier de journée de l'agent recenseur du RGPH 2001, il y aurait 1070 habitants à Gari n kawa et 3123 à Sabon Gari, auxquels s'ajoutent 653 militaires et 439 «visiteurs », ce qui fait une population totale de Dirkou évaluée à 4846 habitants. D'après le Chef de Canton du canton de Kawar (où se trouve Dirkou) "... ces données ne sont pas bonnes, ça a été vraiment mal fait... il y a plein de gens à Dirkou qui n'ont pas été recensés." (Dirkou, le 31octobre 2003). Nos enquêtes de terrain vont dans ce sens et laissent à penser que la population de Dirkou oscille entre 7000 et 10000 personnes. Pourtant, à l'inverse, les résultats définitifs du RGPH 2001, publiés en décembre 2004, indiquent que la population totale de Dirkou est de 2156 personnes, sans détailler la répartition par quartier.
} 
Une foule cosmopolite composée de Nord Africains, de Sahariens et de Subsahariens s'y côtoie ; les passages répétés des individus mobiles (migrants, transporteurs...) y rythment la vie des «immobiles» (commerçants, fonctionnaires...). À l'origine simple point de passage, Dirkou est devenu un lieu d'accueil, de commerce et de rencontre, un lieu de contrôle et d'attentes interminables, de peurs et de tensions également, mais rarement de renoncement.

"Les policiers, quand ils arrivent à Dirkou, ils viennent avec juste une natte sous le bras. Mais quand ils repartent quelques mois plus tard, ils ont des matelas, des télés, des paraboles..."

Propos d'un villageois - Dirkou, mai 2003.

En arrivant dans l'oasis, après avoir traversé le Ténéré, les migrants sont de nouveau taxés par des militaires, par des gendarmes (1 000 à 3000 FCFA par personne), parfois avec violence, puis par des policiers (1000 à 10000 FCFA). Ces taxes arbitraires et surabondantes grèvent le budget des migrants qui, sans argent après avoir tout (et trop) donné aux forces de l'ordre, ne peuvent plus ni faire demi-tour ni continuer leur voyage, et restent parfois des mois avant de pouvoir repartir.

"C'est parce qu'on nous a pris tout notre argent. En arrivant ici à Dirkou on a payé 2000 FCFA. Puis on nous a dit d'entrer dans la gendarmerie [commissariat de police], là on nous a demandé 5000 . Moi j'ai dit je n'ai pas 5000 . C'était 16 heures, à 19 heures j'étais encore là. Ils ont dit 5000 c'est dernier prix. J'ai dormis là, c'est la première fois que je passe une nuit à la police, la première fois. Le matin un autre est venu nous voir, il nous a dit d'amener 1000 francs 1000 francs, on lui a donné et on est sorti. [...] Vraiment l'aventure c'est pas facile... quand j'ai quitté Cotonou je ne savais pas que c'était comme ça... si on m'avait dit que c'était autant de souffrance je ne serais pas parti, je serais resté chez mes parents ; là-bas j'étais bien, je ne manquais de rien, mais ici... ah... C'est la première fois que je viens au Niger, dans le désert comme ça, mais c'est aussi la dernière... la première et la dernière fois, je le jure devant Dieu... même au retour de la Libye, je vais rentrer par vol, si je peux... je ne veux jamais revenir ici..." Yemalo Fêmi "Pedro", Béninois - Dirkou, le 20 décembre 2004. 
De nombreux migrants sont ainsi bloqués un temps sur les routes migratoires nigériennes, faute de moyen suffisant pour poursuivre leur voyage. Il s'agit généralement d'individus ayant prévu de travailler en Libye et qui, partis en migration avec un capital variant entre une centaine de milliers de francs et quelques centaines de milliers de francs CFA selon le pays d'origine, ne peuvent rien faire face aux nombreuses dépenses qu'occasionne la traversée du Niger (ceux dont l'objectif de départ est de se rendre directement en Europe, sans faire escale en Libye, partent généralement avec un budget plus important). Sans possibilité d'aller plus avant ni de retourner chez eux, ces migrants peuvent rester plusieurs semaines, voire plusieurs mois, là où leur voyage a momentanément pris fin, dans l'attente d'une hypothétique aide de leur famille ou à la recherche d'un travail.

Cette situation trouve son origine non seulement dans le décalage qui existe entre le budget prévu par ces candidats à la migration et le coût réel de leur aventure migratoire, mais également dans le fait que les agents de contrôle et de répression de l'Etat taxent systématiquement les migrants, quelle que soit leur capacité financière, au point parfois de les laisser totalement démunis au sortir d'un contrôle.

"Dans ce pays, tous les gens sont pauvres, les ressortissants de ce pays souffrent, donc quand ils voient des étrangers avec un peu d'argent, ils trouvent toutes les possibilités pour le leur prendre... quand ils voient des étrangers ici, ils savent qu'on va en Libye, ils savent qu'on a de l'argent avec nous, et pas seulement un peu d'argent. Ils font tout pour nous le prendre, ils nous ont même frappé à Dirkou parce qu'on ne voulait pas payer... Ils avaient pris nos passeports Ecowas [carnet de voyage CEDEAO des pays anglophones], ils disaient qu'on était en situation illégale parce qu'on n'avait pas de visa... c'est pour ça qu'ils demandaient l'argent, alors qu'avec les passeports Ecowas on peut venir au Niger légalement. [...]. Finalement on a payé pour qu'ils nous redonnent nos passeports, on n'avait pas de choix. Mais il y en a deux avec nous qui ne pouvaient vraiment pas payer, ils ont dû laisser leurs passeports à la police à Dirkou, et ils sont venus ici pour travailler. [...]. Et regarde maintenant, on est là, on est sale, on mange mal, on cuisine avec du bois, on habite dans une maison qui est complètement abîmée... ce n'est même pas une maison... c'est dur, vraiment, mais c'est une expérience, ce qu'on vit ici c'est une grande expérience... quand on rentrera au Nigeria, on aura des histoires à raconter, on pourrait même écrire un livre, chacun de nous pourrait écrire un livre..."

Ibrahim ${ }^{8}$, Nigérian - Bilma, le 8 décembre 2004.

\footnotetext{
${ }^{8}$ Ce migrant nigérian catholique se fait appeler Ibrahim depuis qu'il est arrivé au Kawar afin d'être plus facilement employé par les populations locales musulmanes.
} 
Leurs conditions de vie difficiles, tant moralement que physiquement, les poussent à accepter tout travail qui peut leur permettre de gagner les quelques dizaines de milliers de francs CFA dont ils ont besoin pour atteindre le Fezzan libyen. Ces migrants, étrangers pour la plupart, participent à un changement sur le marché local du travail en proposant une main d'œuvre peu onéreuse et facilement exploitable. C'est ainsi que certains, tel Ibrahim cité ci-dessus, vont travailler aux salines de Bilma à une quarantaine de kilomètres de Dirkou, d'autres restent à Dirkou et travaillent pour des salaires de misère dans n'importe quel domaine, du chargement des camions à la prostitution plus ou moins occasionnelle, du concassage de blocs de calcaire pour la fabrication de briques aux emplois domestiques chez des notables locaux. Dans ce dernier cas, les employeurs sont généralement des fonctionnaires ou d'importants transporteurs, qui ne rémunèrent pas les migrants qu'ils emploient mais les logent et les nourrissent et/ou leur promettent en contrepartie de les envoyer gratuitement en Libye après quelques mois de travail. Ces engagements sont généralement tenus, mais lorsqu'il s'agit de représentants de l'Etat, l'envoi non payant de migrants se fait au détriment des transporteurs qui se voient régulièrement imposer ce type de passagers sans être défrayés.

"Ca fait un mois que je suis là, je n'ai pas l'argent pour aller en Libye, sur la route on m'a tout pris, ils te prennent 1000 FCFA ici, 5000 là, maintenant je n'ai plus rien... je suis venu avec des amis, eux ils ont continué jusqu'en Libye... ici il n'y a rien à faire... je travaille ici [chez un policier] parce que si tu ne fais rien c'est pas bon... mais ça ne va pas, ils me demandent trop de choses, tout le temps, et ils ne me paient pas, ils me donnent seulement de quoi manger... en plus ils m'appellent Christopher je ne sais pas pourquoi, mon nom c'est Hamza..."

Hamza, Nigérian - Dirkou, le 24 décembre 2004.

\section{Les zones militarisées du Nord Niger : dernière étape avant la Libye}

Les gendarmes du détachement de gendarmerie de Dirkou essaient de contrôler les véhicules au moment de leur départ de l'oasis afin de prélever une taxe de 1000 FCFA par migrant en partance. Cette taxe, payée par les chauffeurs des véhicules, est systématiquement négociée, les chauffeurs minimisant le nombre de migrants qu'ils transportent. De leur côté, les gendarmes peuvent menacer d'immobiliser le véhicule s'ils considèrent que le chauffeur n'accepte pas de payer suffisamment. Un accord à l'amiable est toujours trouvé et, si l'argent est donné, c'est le signal du départ.

Aux passages de Ségédine (village où se trouve un groupement des Forces Nigériennes d'Intervention et de Sécurité), de Dao Timi (compagnie militaire) et de Madama (compagnie militaire), les migrants sont encore délestés de quelques milliers de francs $\mathrm{CFA}^{9}$. En cas de refus ou d'incapacité de paiement, ils peuvent être regroupés au soleil en saison chaude, ou arrosés la nuit en saison froide, jusqu'à ce qu'une somme considérée

\footnotetext{
${ }^{9}$ En 2004, un détachement du groupement FNIS de Ségédine a été installé à Aney (oasis situées entre Dirkou et Ségédine), officiellement pour sécuriser la zone, en fait pour en faire un point de contrôle supplémentaire.
} 
comme suffisante par les militaires soit rassemblée. Les transporteurs paient également une taxe de passage non-officielle à chacun de ces points de contrôle militaire. Un chauffeur de camion libyen dit payer aux autorités nigériennes environ 300000 FCFA lorsqu'il va d'Agadez en Libye, auxquels s'ajoutent 1000 FCFA par passager transporté. Les taxes des pick-up sont moindres, de l'ordre d'une centaine de milliers de francs CFA. Les moyens de transport immatriculés au Niger sont semble-t-il moins taxés sur la partie nigérienne du parcours, mais ne peuvent se rendre en Libye; c'est pourquoi certains transporteurs possèdent, pour chacun de leurs véhicules, des plaques d'immatriculation et des papiers des deux pays.

Jusqu'au début des années 2000 les départs de Dirkou en direction de la Libye se sont fait au gré des "fermetures" temporaires de la frontière libyenne. Le Nord du Niger, espace de transit, fonctionnait alors véritablement comme un espace tampon entre les pays de départ et de destinations des migrants. Cette alternance de périodes au cours desquelles le passage par la voie officielle de migrants sans papier était toléré et d'autres où il était nécessaire d'attendre (ou de passer par d'autres voies) a impulsé le développement de deux types de réseaux de transport transfrontaliers. Certains reposent sur le capital social de leurs membres, sur leurs relations avec les représentants des deux Etats dans la zone frontalière, et peuvent ainsi utiliser la voie officielle et passer par les points de contrôle sans que leur activité, bien qu'illégale, soit compromise. D'autres au contraire intègrent un nombre de personnes beaucoup plus restreint et développent une activité clandestine basée sur une connaissance fine des itinéraires de contournement des postes de contrôle

Suite au rapprochement de la Libye et de l'Union Européenne sur la question du contrôle des flux migratoires ${ }^{10}$, la tolérance des autorités libyennes en ce qui concerne l'entrée sur leur territoire de migrants en situation irrégulière est moindre. Les chauffeurs des véhicules qui passent par la voie officielle, c'est-à-dire par les postes frontières, n'acceptent plus de prendre de passagers qui ne peuvent entrer légalement en Libye. De ce fait, le passage illégal de la frontière par les migrants puis leur entrée dans les villes du Sud libyen se réalisent de plus en plus avec discrétion et par les voies détournées de la fraude. ${ }^{11}$

\footnotetext{
10 "C'est à la demande de l'Italie que les Vingt-Cinq ont finalement décidé de lever l'embargo sur les armes [à l'encontre de la Libye]. Rome, qui fait face à un flot de réfugiés tentant de rejoindre ses côtes par la mer, vient en effet de conclure un accord avec Tripoli sur la lutte contre l'immigration clandestine. Ce plan suppose que la Libye soit dotée des équipements - avions, hélicoptères et vedettes rapides notamment - qui lui permettront d'assurer la surveillance et le contrôle des flux migratoires. Les Vingt-Cinq estiment que la coopération avec la Libye sur l'immigration est "essentielle et urgente" et appellent Tripoli à mener "une action effective" contre l'immigration illégale." (Le Monde, 12.10.2004).

${ }^{11}$ Nous ne pourrons aborder ici la question de l'organisation des retours de migrants subsahariens qui, après avoir travaillé quelques temps en Libye, rentrent dans leurs pays d'origine.
} 


\section{DES ACTEURS ENTRE LÉGALITÉ ET LÉGITIMITÉ}

«la fraude n'est pas considérée par la société nigérienne comme étant un délit. » Directeur Général Adjoint de la Douane nigérienne, novembre 1999.

De hauts lieux du transit migratoire émergent au Sahara là où les acteurs institutionnels et les acteurs privés du transport officiel et officieux mettent en place des stratégies utilisant les vides laissés par les Etats pour arriver à leurs fins. Ces lieux spécifiques sont utilisés comme points de contact et d'entendement entre les pouvoirs publics, aux exigences fluctuantes, les acteurs privés, aux pratiques fluctuantes, et les migrants. Chaque passage, chaque contact entre ces sphères, est l'occasion d'une nouvelle discussion qui permet de s'entendre sur les modalités de circulation des individus et donc de celle qui lui est liée, la circulation d'argent. Lors de ces discussions chacun joue sur la légitimité de ses actes pour convaincre l'autre, sans recourir ou presque à la législation en vigueur.

Au Niger plus qu'en Libye, l'approche des pratiques d'acteurs selon leur plus ou moins grande légitimité permet de mieux comprendre les logiques d'action et la manière dont leur cadre de référence s'éloigne progressivement du cadre législatif des Etats.

\section{Les États : laxistes ou "contrebandiers"?}

Un protocole de libre circulation des individus au sein de l'espace CEDEAO existe depuis 1979 (protocole de Dakar), mais il ne s'applique qu'aux ressortissants des pays membres de la CEDEAO et ne concerne donc qu'une partie des migrants sur une partie de leur parcours migratoire. Pourtant, la plupart des migrants subsahariens, quel que soit leur pays d'origine, affirment que, pour voyager d'un pays à l'autre, ils n'ont "pas besoin de visa", qu'"entre Africains, [le passeport] ne sert à rien, il suffit d'une carte d'identité". Ces propos s'expliquent par le fait que l'absence de document de voyage n'empêche effectivement pas la circulation des individus dans cette partie du continent africain et que la possession de papiers officiels, comme les passeports ou les carnets de voyage CEDEAO, ne protège pas les migrants contre les taxes illégales des forces de l'ordre (cf. entretien avec Ibrahim).

"Les interprétations abusives de dispositions réglementaires, elles-mêmes abusives par rapport au texte législatif initial, foisonnent. Les contrôles, qui ne sont pas vraiment des contrôles mais des opportunités de bakchich, se multiplient. Trop souvent, certains membres des administrations douanières et des forces de l'ordre agissent pour leur propre compte et abusent de la situation."12

Que les migrants et les transporteurs soient en situation régulière ou non au regard de la loi nigérienne, ils sont à même de circuler librement sur le territoire national du moment qu'ils paient un certain nombre de taxes arbitraires aux agents de l'Etat qui agissent impunément dans l'illégalité, comme le concède un officier de police

${ }^{12}$ Conseil Nigérien des Utilisateurs des Transports publics, 2002, Séminaire de formation sur la fiscalité au Niger-23-24 octobre 2002, Niamey : 91. 
de Dirkou : "Vous savez, ici on est au désert, loin de tout. Moi, mon travail ici c'est d'être chef d'autogare, c'est tout ce qu'il y a à faire. Tous on prend l'argent, on est tous dans l'illégalité, mais c'est comme ça, qu'est-ce qu'on y peut..." (Dirkou, mai 2003).

Du côté libyen, la politique migratoire de l'Etat rend la situation sensiblement différente, mais tout aussi ambiguë. La libre circulation des individus entre Etats d'Afrique de l'Ouest et du Nord, évoquée dans la charte de la CEN-SAD de 1998, n'a jusqu'à ce jour rien d'officielle. Pourtant, l'immigration des Subsahariens est alternativement tolérée (et même incitée) par les dirigeants libyens, et interdite voire combattue, comme en témoignent les expulsions manu militari d'immigrants ${ }^{13}$, dont l'une des dernières vagues fit dire à un journaliste nigérien que "cette expulsion radicale des Africains subsahariens pose de sérieuses interrogations sur le sens que donne Kadhafi à l'Union Africaine (UA) et à la Communauté des Etats Sahélo-Sahariens (CEN-SAD)." ${ }^{14}$ Ce côté officieux de l'immigration semble maintenu plus ou moins volontairement par les dirigeants libyens, afin de contenir la masse des migrants dans le Sud du pays (où ils sont employés aux travaux agricoles, de construction...), et de conserver le caractère réversible de ces mouvements de populations par la pratique des expulsions massives.

Les formes de contrôle et de taxation des individus mobiles (migrants et transporteurs) par les agents des Etats nigérien et libyen, le long de l'axe Agadez-Dirkou-Sebha, l'officialisation au Niger des agences de transport qui permettent une émigration illicite vers les pays d'Afrique du Nord ou encore les appels à l'immigration de travailleurs africains du colonel Kadhafi, suivis d'expulsions de ces mêmes immigrants, sont autant de faits qui mettent en évidence l'ambiguïté des positions des Etats face à ces circulations humaines transsahariennes. Ce qui nous renvoie au questionnement de Roger Botte dans son introduction au numéro de Politique africaine consacré à la globalisation et à l'illicite en Afrique: "Les activités trafiquantes aujourd'hui à l'œuvre sur le continent sont-elles l'expression d'un état d'anomie et de faillite des Etats ou bien constituent-elles une espèce d'aggiornamento du politique après plus de vingt ans de conditionnalités néolibérales plus ou moins dogmatiques." (Botte, 2004 : 8).

"Policiers, gendarmes, douaniers, tout ça c'est la mafia, ils sont tous coxers !" Adou, passeur, Agadez, avril 2003.

\footnotetext{
13 «...pour la seule année 2004, les autorités libyennes estiment à 54000 le nombre des personnes "rapatriées". En 2003, le nombre officiel des expulsés était de 43 000. » (Le Monde, 05.06.05).

${ }^{14}$ Le Républicain [2004], "Près de 5000 Nigériens expulsés de Libye - Calvaire à travers le désert", $n^{\circ} 637,7-13$ octobre, Niamey : 5.
} 
La législation nigérienne en matière de transport et d'immigration n'est pas appliquée dans une grande partie du pays : en permanence, les acteurs du système migratoire saharien sont amenés à négocier entre eux les modalités de leur mobilité ou d'exercice de leur activité, selon une légitimité des actes auto-déterminée bien plus que selon leur légalité. Ce désengagement apparent de l'Etat renvoie, tout à la fois, au laxisme des dirigeants politiques et au dysfonctionnement des structures étatiques qui ne peuvent asseoir leur pouvoir sur l'ensemble du territoire national, et à une évolution du fonctionnement d'un Etat qui tend à se criminaliser ${ }^{15}$. Celui du Niger dont les agents agissent hors du contrôle ou sans l'aval de leurs structures de tutelles encourage implicitement leur fraude en les rémunérant peu, en ne contrôlant pas leur travail et en ne prenant aucune mesure pour limiter et condamner les abus de pouvoir auxquels ils se livrent. On assiste donc à un glissement complice de l'Etat et de ses agents dans un processus d'informalisation des pratiques (voire de criminalisation lorsque celles-ci ne sont plus légitimes aux yeux de la majorité de la population) et d'indifférenciation partielle des activités légales et illégales.

Face à ces pratiques frauduleuses ou aux injonctions des Etats, des groupes sociaux s'organisent et produisent des stratégies de contournement qui évoluent selon des normes officieuses et insoumises aux politiques étatiques (cf. Tidjani Alou, 2004). Tel est le cas des acteurs privés du système migratoire saharien qui ont mis en place depuis le début des années 2000 des réseaux de transport clandestins au Sahel afin de limiter le coût financier et temporel des multiples contrôles des représentants de l'Etat nigérien et l'impact des "fermetures" de plus en plus fréquentes de la frontière libyenne ${ }^{16}$.

\section{Quelle quantification possible des flux migratoires transsahariens?}

Les flux migratoires transsahariens, officieux, illégaux et de plus en plus fréquemment clandestins, sont difficilement quantifiables. Pour ce qui est des circulations migratoires passant par la voie officielle au Niger, bien qu'aucune étude spécifique n'ait été réalisée et que les recensements généraux de la population n'en parlent pas ${ }^{17}$, des indicateurs bruts existent. Il s'agit des taxes prélevées par les services préfectoraux de Bilma sur le "transit exodant" et sur les "véhicules de transport", des taxes du syndicat des transporteurs sur le transport de passagers, et du contrôle des entrées et des sorties du territoire national par les forces de l'ordre (police et gendarmerie). L'étude des carnets des taxes préfectorales et syndicales met en évidence une grande irrégularité

\footnotetext{
${ }^{15}$ Cf. Bayart, Ellis et Hibou [1997].

${ }^{16}$ Ces "fermetures" de frontières sont parfois sélectives et peuvent ne concerner que les migrants anglophones ou bien uniquement les migrants non-sahéliens, ou, comme c'est le cas depuis le début de l'année 2005, concerner l'ensemble des migrants ressortissants de pays non limitrophes de la Libye.

17 Lors du recensement de 1988, «[...] aucune question n'a été posée sur l'émigration, c'est-à-dire sur les mouvements des populations du Niger vers d'autres pays » (Ministère de l'Economie et des Finances, 1992 : 30). Pour le recensement de 2001, «il est impossible d'obtenir des données relatives à l'émigration internationale » (Ministère de l'Economie et des Finances, 2004 : 7).
} 
des prélèvements et une approximation systématique du nombre de passagers par véhicule. Le percepteur des impôts de la Préfecture de Bilma reconnaît lui-même à propos des différentes taxes concernant le domaine des transports qu'elles "sont perçues par des collecteurs qui sont dans les villages... quand ils ont fini un carnet ils nous le ramènent et on les paie... mais ce n'est pas toujours régulier, et moi-même je ne sais pas toujours comment ils font leur travail... je ne sais pas trop à quoi correspondent ces taxes." (Bilma, le 6.12.2004). En outre, de nombreux chauffeurs et transporteurs évitent les lieux où ces taxes sont prélevées. "On ne peut pas les forcer à payer" résume un membre du syndicat des transporteurs, avant de poursuivre en expliquant que "par exemple sur soixante camions qui arrivent à Dirkou, il y en a peut-être vingt qui vont s'arrêter ici à l'autogare... vraiment c'est pas facile de savoir combien de véhicules arrivent ici, c'est pour ça qu'on a déposé les registres, ça ne servait à rien." (Dirkou, le 21.12.2004).

Les données de la gendarmerie concernant les mouvements des personnes et des véhicules entre Agadez et la Libye (fig. 3) sont également très approximatives. Les rares chiffres disponibles sont à regarder à l'aune des propos du lieutenant de gendarmerie de Dirkou qui explique que "pour les passagers qui viennent ici, c'est vrai que les chauffeurs nous donnent des listes, mais une fois qu'on a compté les passagers des véhicules, on ne les garde pas, on les jette... et puis il y a trop de véhicules qui nous contournent... c'est pour ça 
qu'on ne fait pas de statistiques... vraiment c'est pas facile de savoir combien il y a d'exodants qui viennent ici." (Dirkou, décembre 2004). Le constat du commandant de la Brigade de Gendarmerie de Bilma concernant leurs statistiques va dans le même sens : "Et puis vous savez, ici, ce n'est pas facile de contrôler tout le monde, il y a beaucoup de véhicules qui nous contournent. Les chiffres qu'on a ne sont pas à la virgule près, c'est vraiment approximatif." (Dirkou, le 21.12.2004).

Fig. 3 - Mouvements des véhicules et des personnes sur l'axe Agadez-Dirkou-Libye

\begin{tabular}{|c|c|c|c|c|c|c|c|c|}
\multicolumn{1}{c|}{} & \multicolumn{3}{c}{1993} & \multicolumn{2}{c|}{1994} & \multicolumn{3}{c|}{2001} \\
\cline { 2 - 10 } \multicolumn{1}{c|}{} & Toyota* & Camions & Personnes & Toyota* & Camions & Toyota* & Camions & Personnes \\
\hline Agadez-Dirkou & - & 4 & 15 & 13 & 382 & 12 & 510 & 4242 \\
\hline Dirkou-Libye & 5 & 5 & 165 & 234 & 451 & 184 & 530 & 5835 \\
\hline Libye-Dirkou & 263 & 407 & 6962 & 408 & 667 & 220 & 573 & 8488 \\
\hline Dirkou-Agadez & 211 & 376 & 6575 & 27 & 92 & 70 & 662 & 20059 \\
\hline TOTAL & 479 & 792 & 13717 & 682 & 1592 & 486 & 2275 & 38624 \\
\hline
\end{tabular}

* désigne les voitures tout terrain à plateau.

Source : Poste de gendarmerie de Dirkou (2003)

Les statistiques du poste de police frontalier de Dirkou (fig. 4 et 5) semblent corroborer certains de nos propos. La forte diminution entre 2003 et 2004 des sorties du territoire en direction de la Libye (fig. 4) peut par exemple renforcer à la fois l'idée d'un durcissement de la politique migratoire libyenne et celle du développement de réseaux de transport clandestins qui évitent les postes frontaliers. Mais certains signes, tels que l'absence totale de ressortissants du Nigeria dans les sorties du territoire comptabilisées en 2002 (fig. 5), laissent dubitatif et rappellent que les méthodes de dénombrement utilisées ne sont pas fiables.

Fig. 4 - Mouvement des personnes entre le Niger et la Libye - poste de police frontalier de Dirkou (2003-2004)

\begin{tabular}{|c|r|r|}
\cline { 2 - 3 } \multicolumn{1}{c|}{} & \multicolumn{1}{c|}{2003} & 2004 \\
\hline Nombre total des personnes entrant au Niger & 24854 & 24412 \\
\hline Nombre de Nigériens entrant & 22737 & 20782 \\
\hline Nombre d'étrangers CEDEAO entrant & 1271 & 1975 \\
\hline Nombre d'étrangers hors CEDEAO entrant & 846 & 1611 \\
\hline Nombre total des personnes sortant du Niger & 14475 & 5649 \\
\hline Nombre de Nigériens sortant & 8437 & 2355 \\
\hline Nombre d'étrangers CEDEAO sortant & 2796 & 1868 \\
\hline Nombre d'étrangers hors CEDEAO sortant & 3242 & 1426 \\
\hline \multicolumn{2}{|c|}{} \\
\hline Personnes refoulées de Libye : Nigériens & 1574 \\
\hline Personnes refoulées de Libye : étrangers & 138 \\
\hline
\end{tabular}

Sources : Poste de police frontalier, Dirkou / Commissariat central, Agadez. 
Fig. 5 - Sorties du Niger en direction de la Libye - poste de police frontalier de Dirkou (2002)

\begin{tabular}{|c|c|}
\hline Nationalités & $\begin{array}{c}\text { Nombre de sorties } \\
\text { en } 2002\end{array}$ \\
\hline Nigériens & 8142 \\
\hline Ghanéens & 1462 \\
\hline Tchadiens & 340 \\
\hline Maliens & 289 \\
\hline Burkinabés & 173 \\
\hline Libyens & 136 \\
\hline Sénégalais & 86 \\
\hline Togolais & 37 \\
\hline Ivoiriens & 31 \\
\hline Guinéens & 27 \\
\hline Béninois & 24 \\
\hline Camerounais & 23 \\
\hline Congolais & 20 \\
\hline Gambiens & 16 \\
\hline Soudanais & 11 \\
\hline Sierra Leonais & 8 \\
\hline Autres & 18 \\
\hline Total & 10843 \\
\hline
\end{tabular}

Source : Direction de la Sécurité du Territoire - Niamey.

Bien que ces données permettent au Ministère de l'Intérieur de tenir des statistiques officielles sur les entrées et sorties du territoire national, le Directeur départemental de la Police Nationale d'Agadez reconnaît que "généralement, si tu vois la personne passer au poste, c'est qu'il est en règle, mais les clandestins, eux, ils passent en douce, et ça dépasse deux fois ces chiffres-là." (Agadez, le 29.12.2004).

Le but premier de ces comptages factices réalisés par la police, comme de ceux des services préfectoraux et du syndicat, est de taxer les migrants et les transporteurs. Aussi, au-delà de la difficulté matérielle à effectuer des comptages précis, se pose la question de la volonté 
des acteurs du contrôle de réaliser un tel travail. Qui trouverait un intérêt à rendre visible la réalité de ces circulations humaines illégales alors que nombre d'arrangements entre acteurs privés et publics, acceptés par l'Etat, sont justement basés sur l'opacité du système. Si, à terme, les pressions exercées par l'Union Européenne sur la Libye, afin de mieux contrôler les flux migratoires en provenance d'Afrique subsaharienne, se reportaient sur le Niger et y trouvaient un écho favorable, au vu du développement rapide des réseaux migratoires clandestins, la question de la quantification de ces flux resterait entière.

Réussir à dénombrer avec précision les flux migratoires transsahariens semble encore improbable, toute extrapolation à partir des données obtenues lors d'observations de terrains restant hasardeuse. Si l'on se prête tout de même à cet exercice en recoupant les quelques données statistiques disponibles dans les services publiques nigériens, les discours de divers acteurs qui relatent une diminution des flux depuis 1999, les chiffres avancés par Ali Bensaad dans son étude sur le rôle d'Agadez dans les migrations transsahariennes [Bensaad, 2002 : 248] et les résultats de nos propres enquêtes, il est simplement possible de parler de quelques dizaines de milliers de migrants transitant annuellement par le Niger en direction de la Libye, dont un à deux tiers essaient ensuite de rejoindre l'Europe.

\section{Les migrations internationales face aux États : réseaux et territoires}

Le renouveau des migrations transsahariennes au début des années 1990 s'est accompagné d'une diversification des formes migratoires. Les réseaux officiels et clandestins qui permettent le passage des migrants d'une rive à l'autre du Sahara, animés par des acteurs aux logiques et aux pratiques fluctuantes, se sont alors développés par l'intégration de nombreux agents de l'Etat issus de différents corps de contrôle et de sécurité. Au Niger, en accord avec l'Etat de façon implicite, ces agents tirent depuis lors profit de circulations humaines illégales.

Ces pratiques des pouvoirs publics ne permettent plus de percevoir clairement la limite entre le licite et l'illicite et renvoient à l'idée de R. Botte selon lequel, dans un Etat illégallégal, « les activités trafiquantes à l'œuvre aujourd'hui sur le continent manifestent [...] l'apparition d'un nouveau type d'accumulation à la fois par l'Etat et par des réseaux à côté de l'Etat (mais non contre lui)» [Botte, op. cit. : 20]. Ce modèle d'accumulation conjointe d'acteurs privés et publics à partir d'activités illégales, valable un temps pour le système migratoire saharien, semble remis en cause par ses évolutions récentes. Les dérives des pratiques des pouvoirs publics, dont la volonté d'accumulation semble excessive, participent en effet indirectement du développement d'activités clandestines qu'ils ne peuvent contrôler et dont ils ne bénéficient aucunement. Cette transformation en cours du système migratoire saharien interroge tant la nature même de l'Etat nigérien que sa capacité à préserver ses intérêts financiers, légaux ou non, et l'intégrité de son territoire. 
Les flux migratoires transsahariens engendrent et impulsent la création de nombreuses infrastructures et activités dans les espaces qui leur servent de relais, générant ainsi l'existence d'un véritable maillage urbain du Sahara central. Ces lieux, carrefour migratoire comme Agadez ou lieu d'étape comme Dirkou, sont mis en relation par les circulations humaines et deviennent les points d'ancrage de territoires réticulaires qui n'existent que par et pour ces circulations. Une étude plus fine de la structuration de ces espaces sahariens permettrait de différencier, d'une part, les constructions spatiales de chacun des groupes d'acteurs intervenant dans le système migratoire, ce qui ferait apparaître en certains endroits un espace « feuilleté », et, d'autre part, les territoires au sein desquels interviennent encore des acteurs publics de ceux dont ils sont totalement absents. Ces territoires de la circulation, où la légitimité d'action prime généralement sur toute forme de légalité, peuvent être qualifiés de «territoires de la négociation » et de plus en plus « de la clandestinité », ce qui dans les deux cas interroge leur rapport à l'ensemble du territoire national tel qu'il est envisagé par l'Etat.

\section{BIBLIOGRAPHIE}

BAYART J.-F. [2004], "Le crime transnational et la formation de l'Etat", Globalisation et illicite en Afrique, Politique africaine (93) : 93-104.

BAYART J.-F., ELLIS S. et HIBOU B. [1997], La Criminalisation de l'État en Afrique, Bruxelles, Complexe.

BENSAAD A. [2002], "Echanges et flux migratoires transsahariens : Agadez, place marchande et carrefour migratoire", in M. Peraldi (dir.), La fin des norias ? Réseaux migrants dans les économies marchandes en Méditerranée, Paris, Maisonneuve \& Larose : 243-267.

BOTTE R. [2004], "Vers un Etat illégal-légal ?", Globalisation et illicite en Afrique, Politique africaine (93) : 7-20.

BOUBAKRI H. [2001], "Le Maghreb et les nouvelles configurations migratoires internationales : mobilité et réseaux régionaux voisins (Europe et Afrique subsaharienne)", www.irmcmaghreb.org, Correspondances (68) : 8-15.

CONOMOS A. [1995], "Sociologie et science politique : les réseaux, théories et objets d'études", Revue Française de Science Politique, 45 (1) : 165-178.

ENOH C., KOFFI E., LABAZEE P. [2001], "Besoin d'Etat, évolution des approches, réponses des acteurs", in G. Winter (coord.), Inégalités et politiques publiques en Afrique : pluralités des normes et jeux d'acteurs en Afrique, Paris, IRD-Karthala : 397-413.

GUITART F. [1992], « Commerce et transport à Agadez au début des années 1970 », in H. D'Almeida-Topor, C. Chanson-Jabeur, M. Lakroum (eds.), Les transports en Afrique $\left(X I X^{e}-X X^{e}\right)$, L'Harmattan, Paris : 247-258.

HADDAD S. [2000], "La politique africaine de la Libye : de la tentation impériale à la stratégie unitaire", Monde arabe, Maghreb-Machrek (170) : 29-38.

Le Républicain [2004], n604, 15-21 janvier, Niamey : 9.

MINISTERE DE L'ECONOMIE ET DES FINANCES [1992], Recensement Général de la Population 1988 - Analyse des données définitives - Migrations, Niamey, Bureau Central du Recensement, p. 71.

MINISTERE DE L'ECONOMIE ET DES FINANCES [2004], Séminaire d'évaluation des rapports provisoires de l'analyse des résultats définitifs du RGP/H 2001 du 21 au 23 décembre 2004, Niamey, Bureau Central du Recensement, p. 39. 
MINISTERE DE L'ECONOMIE ET DES FINANCES, [2004], Recensement Général de la Population et de l'Habitat - RGP/H 2001, Résultats définitifs : répartition par sexe et par groupe d'ages de la population du Niger en 2001, Niamey, Bureau Central du Recensement $256 \mathrm{p}$.

OFFNER J.-M., PUMAIN D. (dir.) [1996], Réseaux et territoire, significations croisées, Paris, Editions de l'Aube, collection Territoire, 284 p.

OLIVIER DE SARDAN J.-P. [2004], "La rigueur du qualitatif. L'anthropologie comme science empirique", EspacesTemps (84-85-86) : 38-50.

PLIEZ O. [2002], "Vieux réseaux et nouvelles circulations entre les deux rives du Sahara, in M. Cote (dir.), "Le Sahara, cette "autre Méditerranée"", Méditerranée, 99 (3-4) : 31-40.

TARRIUS A. [2002], "Au-delà des Etats-nations : sociétés, cultures et réseaux de migrants en Méditerranée occidentale", in M. Peraldi (dir.), La fin des norias ? Réseaux migrants dans les économies marchandes en Méditerranée, Paris, Maisonneuve \& Larose : 479-488. TIDJANI ALOU M. [2004], "Etat, réseaux marchands et mondialisation. Le cas des convois au Niger", in L. Monnier et Y. Droz (dir.), Côté jardin côté cour - Anthropologie de la maison africaine, Nouveaux cahiers de l'IUED, Paris, PUF : 125-140.

\section{Résumé :}

L'axe transsaharien Agadez-Dirkou-Sebha est devenu depuis quelques années le théâtre d'importants mouvements migratoires régionaux et intercontinentaux. Ces circulations humaines, illégales pour la plupart, sont animées par des réseaux migratoires au sein desquels agissent divers groupes d'acteurs publics et privés. L'organisation de ces groupes et les relations qui les lient renseignent sur l'évolution de leurs logiques et montrent comment l'Etat et ses agents tendent à ne plus différencier le licite de l'illicite.

Mots clés :

Sahara, Niger, Libye, Migration, Etat, Réseaux, Corruption, Légalité, Légitimité. 